【研究简报】

\title{
$\mathrm{SrNi}_{x} \mathrm{~V}_{6-x} \mathrm{O}_{11}$ 的合成和晶体结构
}

\author{
刘红超 郭常霖 屠恒勇 \\ (中国科学院上海硅酸盐研究所, 上海 200050)
}

\section{关键词 $\mathrm{SrNi}_{x} \mathrm{~V}_{6-x} \mathrm{O}_{11}$ 晶体结构 $\mathrm{X}$ 射线粉末衍射 Rietveld 方法 键价方法}

$\mathrm{AV}_{6} \mathrm{O}_{11}(\mathrm{~A}$ 为碱金属或碱土金属)型结构化合物令人感兴趣的是其结构上 $3+$ 和 $4+$ 离子 的分布及转换和其性能上表现出的金属导电性及铁磁性 ${ }^{[1-3]}$. 研究这类物质的结构对了解和 改善其性能, 研究材料化学一般规律都有很重要的意义. De Roy 等 ${ }^{[4]}$ 采用电解方法首次合成 $\mathrm{NaV}_{6} \mathrm{O}_{11}$, 并确定了其晶体结构, 但其空间群后来被证实是错误的. Kanke 等 ${ }^{[5]}$ 得到了 $\mathrm{Sr}_{6} \mathrm{O}_{11}$ 及 $\mathrm{V}$ 离子被 $\mathrm{Ti}, \mathrm{Cr}$ 和 $\mathrm{Fe}$ 等部分取代的化合物的晶体结构和 $\mathrm{X}$ 射线粉末衍射数据, 以 求了解不同取代离子对晶体结构和性能的影响; 后来又通过 $\mathrm{SrV}_{6} \mathrm{O}_{11}, \mathrm{NaV}_{6} \mathrm{O}_{11}$ 单晶样品得到 了各自的晶体结构 ${ }^{[6]}$ 。最近, Kanke 等 ${ }^{[7]}$ 通过 $\mathrm{X}$ 射线单晶衍射和中子衍射方法揭示了 $\mathrm{NaV}_{6} \mathrm{O}_{11}$ 的结构相变. 然而,这些工作都没能很好地揭示不同价态金属阳离子在晶格中的分 布情况, 有些结果甚至是相互矛盾的, 如 Kanke 等 ${ }^{[5]}$ 关于 $\mathrm{SrTiV}_{5} \mathrm{O}_{11}$ 中 $\mathrm{M}(3)$ 位 $\mathrm{Ti}$ 离子价态的 描述, 也正如他们所指出的阳离子分布并不都遵循 Madelung 能量法计算的结果 ${ }^{[6]}$.

本文为了进一步研究取代离子对 $\mathrm{AV}_{6} \mathrm{O}_{11}$ 晶体结构的影响, 以高温固相化学反应方法合成 了 $\mathrm{Ni}$ 部分取代 $\mathrm{SrV}_{6} \mathrm{O}_{11}$ 中的 $\mathrm{V}$ 离子的化合物, 由 $\mathrm{X}$ 射线粉末衍射 Rietveld 方法得到了合成物 质的晶体结构, 用晶体化学键价方法计算了不同晶格位的离子价态, 得到更为合理的结果.

\section{1 实验}

\section{1 合成}

$\mathrm{V}_{2} \mathrm{O}_{3}$ 由 $\mathrm{V}_{2} \mathrm{O}_{5}$ 在 $10 \%$ 氢气气氛中, 温度为 $1137 \mathrm{~K}$ 还原得到. $\mathrm{Sr}_{2} \mathrm{~V}_{2} \mathrm{O}_{7}$ 是 $\mathrm{SrCO}_{3}$ 和 $\mathrm{V}_{2} \mathrm{O}_{5}$ 在空气中, $1137 \mathrm{~K}$ 反应制得. $\mathrm{SrNi}_{x} \mathrm{~V}_{6-} \mathrm{O}_{11}(x=0.10)$ 由 $\mathrm{Sr}_{2} \mathrm{~V}_{2} \mathrm{O}_{7}, \mathrm{Ni}_{2} \mathrm{O}_{3}$ 和 $\mathrm{V}_{2} \mathrm{O}_{3}$ 按一定比 例混合后, 放入 $\mathrm{N}_{2}$ 气氛中, 于 $1337 \mathrm{~K}$ 反应得到. 所有产物均经过 $\mathrm{X}$ 射线粉末衍射鉴定无异 相存在.

\section{$1.2 \mathrm{X}$ 射线粉末衍射}

将产物研成粉末, 在 RIGAKU D/max III B 衍射仪上收集 X 射线粉末衍射数据. 环境温 度为 $(20 \pm 1)^{2} \mathrm{C}$. 仪器条件为: 铜靶, $40 \mathrm{kV}, 25 \mathrm{~mA}$, 石墨单色器, 步进扫描 $0.04^{\circ}(2 \theta) /$ 步, 计 数时间为 $8 \mathrm{~s} /$ 步. 衍射峰位以高纯硅粉的衍射谱来校正.

\section{2 结果与讨论}

\section{$2.1 \mathrm{X}$ 射线粉末衍射图谱指标化及晶体结构精信}

用 TREOR90 程序对衍射图谱进行指标化, 属六角晶系, $F_{20}=50.9(0.018,22)$. 晶胞参 数为: $a_{0}=0.57708(8) \mathrm{nm}, c_{0}=1.3084(2) \mathrm{nm} . X$ 射线粉末衍射图谱参见图 1. 通过消光 规律分析可知其空间群仍为 $\mathrm{P}_{3} / \mathrm{mmc}$. 


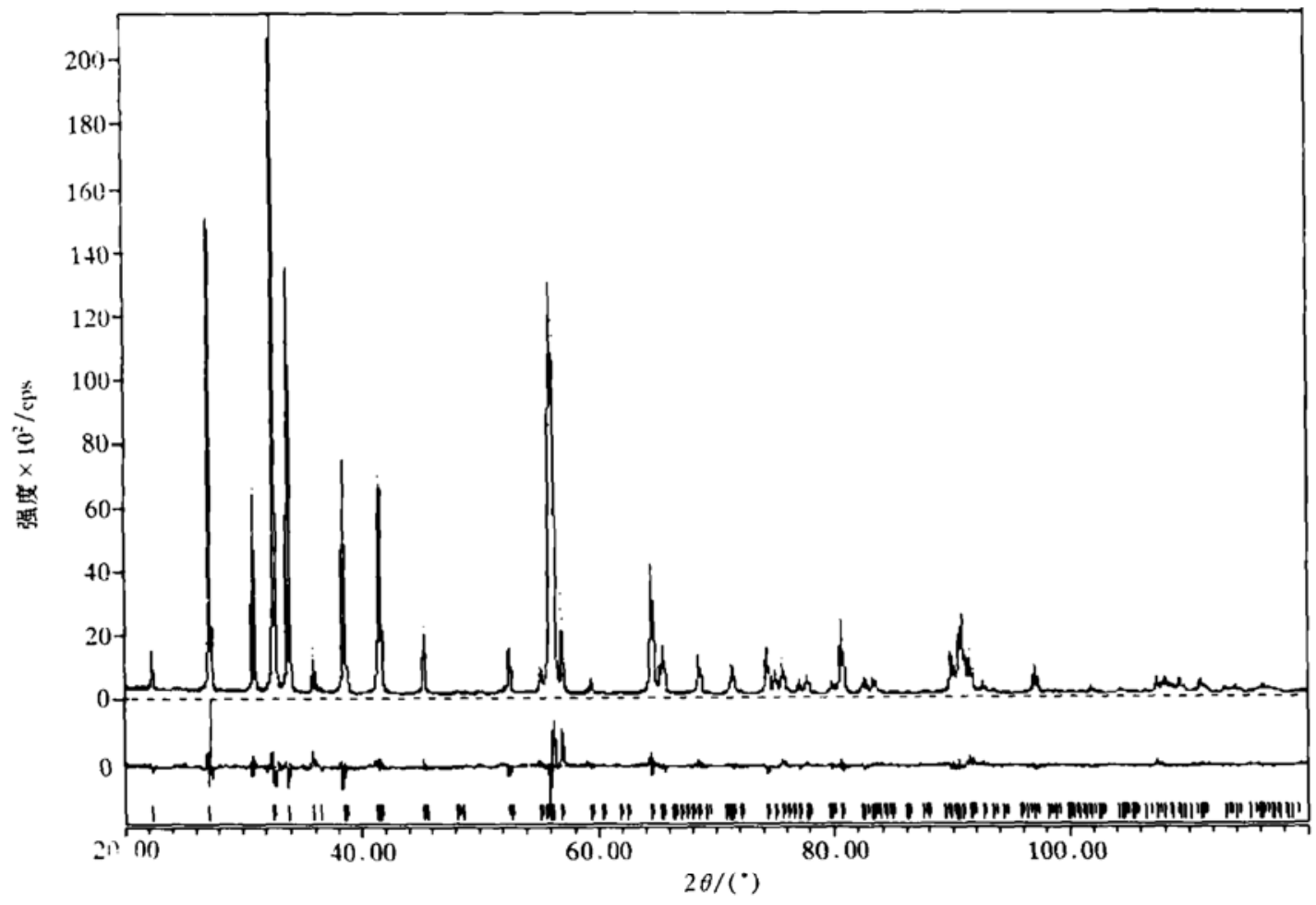

图 $1 \mathrm{SrNi}_{x} \mathrm{~V}_{6-x} \mathrm{O}_{11}(x=0.1)$ 的衍射图谱

点线为实验值, 实线为计算值, 图下方竖直短线为衍射峰位, 曲线为计算值和实验值的偏差

Rietveld 方法的计算程序采用 DBWS9411 程序, 对仪器及样品特征参数、晶胞参数、峰形 参数、结构参数等共 40 个参数进行精修. 先将 $\mathrm{SrV}_{6} \mathrm{O}_{11}$ 的晶体结构参数作为初始参数, 所有 可变参数都参与修正, 得到 $R_{\mathrm{p}}$ 为 $10.2 \%, R_{\mathrm{wp}}$ 为 $13.1 \%$. 在相应 $\mathrm{M}(1), \mathrm{M}(2)$ 和 $\mathrm{M}(3)$ 位加入 $\mathrm{Ni}$ 离子, 继续进行结构精修, 得到 $\mathrm{M}(1)$ 和 $\mathrm{M}(2)$ 位 $\mathrm{Ni}$ 离子占有率为负值; 因此, 在后续的精修 过程中, 只保留 $\mathrm{M}(3)$ 位的 $\mathrm{Ni}$ 离子(任何将 $\mathrm{Ni}$ 置于其他位置的努力都不成功). 由此可见, $\mathrm{Ni}$ 优先进入 $\mathrm{M}(3)$ 位, 这与 Kanke 等 ${ }^{[5]}$ 所得到 $\mathrm{SrFeV}_{5} \mathrm{O}_{11}$ 中 $\mathrm{Fe}$ 在 $\mathrm{M}(3)$ 位占有率较大是一致的. 最后进行择优取向校正, 方向为 $(001)$, 择优取向参数为 0.743 . 最终得到 $R_{\mathrm{p}}=9.0 \%, R_{\mathrm{wp}}=$ $12.2 \%$, 结果列入表 $1 . M(1)$ 位和 $M(2)$ 位温度因子值比通常由 $X$ 射线粉末衍射所得到的值 小, 这与精修过程中仅采用 $\mathrm{V}^{3+}$ 的散射系数有关. 由晶体结构所得的计算 $\mathrm{X}$ 射线衍射谱和实 验谱图示于图 1.

表 $1 \mathrm{SrNi}_{x} \mathrm{~V}_{6-} \mathrm{O}_{11}(x=0.1)$ 的晶体结构参数 (括号中为标准偏差) ) $^{\mathrm{a}}$ )

\begin{tabular}{cclccc}
\hline 原子 & 晶格位 & $x$ & $y$ & $z$ & $B_{\text {iso }} \times 10^{-2} / \mathrm{nm}^{2}$ \\
\hline $\mathrm{Sr}$ & $2(\mathrm{c})$ & $1 / 3$ & $2 / 3$ & $1 / 4$ & $0.9(1)$ \\
$\mathrm{M}(1)$ & $6(\mathrm{~g})$ & $1 / 2$ & 0 & 0 & $0.30(5)$ \\
$\mathrm{M}(2)$ & $4(\mathrm{e})$ & 0 & 0 & $0.1461(2)$ & $0.20(6)$ \\
$\mathrm{M}(3)$ & $2(\mathrm{~d})$ & $1 / 3$ & $2 / 3$ & $3 / 4$ & $0.7(2)$ \\
$\mathrm{O}(1)$ & $12(\mathrm{k})$ & $0.1730(2)$ & $0.3460(2)$ & $0.0777(5)$ & $0.43(1)$ \\
$\mathrm{O}(2)$ & $6(\mathrm{~h})$ & $0.1419(2)$ & $0.2839(2)$ & $3 / 4$ & $0.8(3)$ \\
$\mathrm{O}(3)$ & $4(\mathrm{f})$ & $1 / 3$ & $2 / 3$ & $0.5921(8)$ & $0.56(1)$ \\
\hline
\end{tabular}

a) 空间群: $\mathrm{P} 6_{3} / \mathrm{mmc}$, 晶胞参数: $a_{0}=0.57708(8) \mathrm{nm}, c_{0}=1.3084(2) \mathrm{nm}$, 单胞中分子数: $Z=2$, 计算密度: $D_{x}=4.869$ $\mathrm{g} / \mathrm{cm}^{3}$ 


\section{2 晶体结构}

$\mathrm{SrNi}_{x} \mathrm{~V}_{6-} \mathrm{O}_{11}(x=0.1)$ 的晶体结构和 $\mathrm{SrV}_{6} \mathrm{O}_{11}$ 相比, 晶胞参数中的 $C$ 轴略有拉长, 这主 要是因为 $\mathrm{Ni}$ 离子的半径较 $\mathrm{V}$ 的大, 而取代的量又小; $\mathrm{M}$ 位金属离子和氧间键长、键角也有改 变, 其中以 $\mathrm{M}(3)$ 位变化最大, $\mathrm{M}(3)$ 的化学键变弱, 参见表 $2 . \mathrm{SrNi}_{x} \mathrm{~V}_{6-} \mathrm{O}_{11}$ 的晶胞和沿 $(001)$ 向投影图示于图 2. $\mathrm{M}$ 与 $\mathrm{O}$ 的成键状况在图中已示出. 其晶体结构可描述为 $\mathrm{O}$ 离子沿 (001) 方向的层状六方密堆积, 每三层中有一层的 1/4 O 原子由 $\mathrm{Sr}$ 取代. 由图 2 可以看出, $\mathrm{SrNi}_{x} \mathrm{~V}_{6-} \mathrm{O}_{11}$ 晶胞中有 3 个 $\mathrm{V}$ 位置: $\mathrm{V}(1)$ 处于两个 $\mathrm{O}(3)$ 和 4 个 $\mathrm{O}(1)$ 所构成的、稍有变形八 面体的中心位置, 上下两个 $O(3)$ 与 $V(1), O(1)$ 平面所形成的角度分别为 $93.11^{\circ}$ 和 $86.99^{\circ}$. 这些原子构成连接紧密的网络状结构, 形成的面垂直于 $C$ 轴. 该位置原子排列整齐, 不易被 取代, 因此, 通常情况下, 该位置的取代原子占有率较小. $V(2)$ 位于平行 $C$ 轴的棱上、 $O(1)$ 和 $\mathrm{O}(2)$ 所形成的三棱柱中心, 与另一个 $\mathrm{V}(2)$ 的三棱柱共 $\mathrm{O}(2)$ 面. $\mathrm{V}(3)$ 和部分进入该位置的 $\mathrm{Ni}$

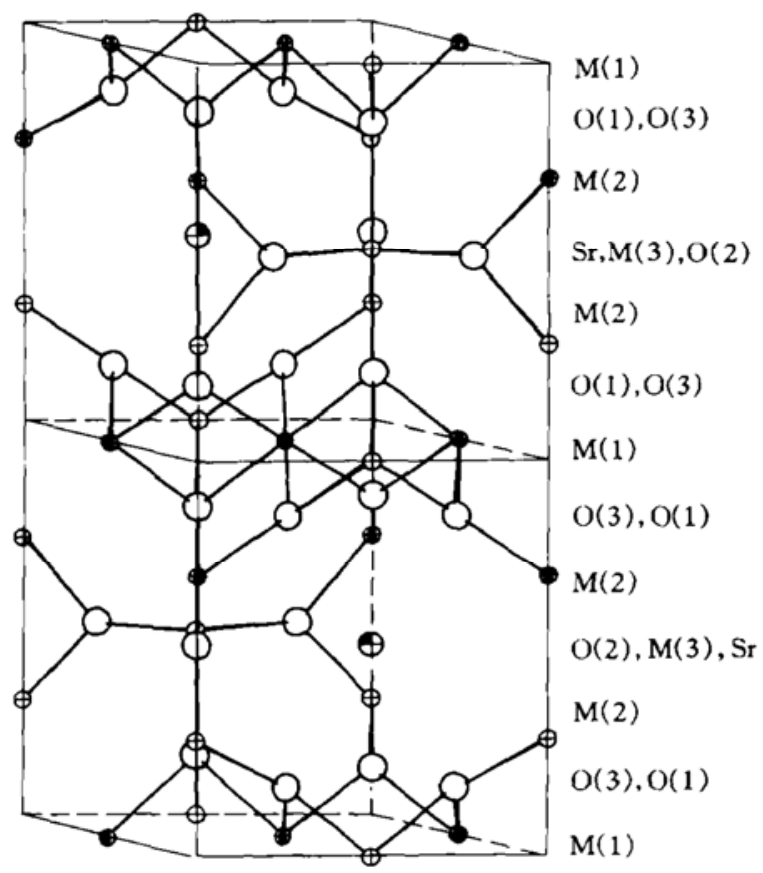

(a)

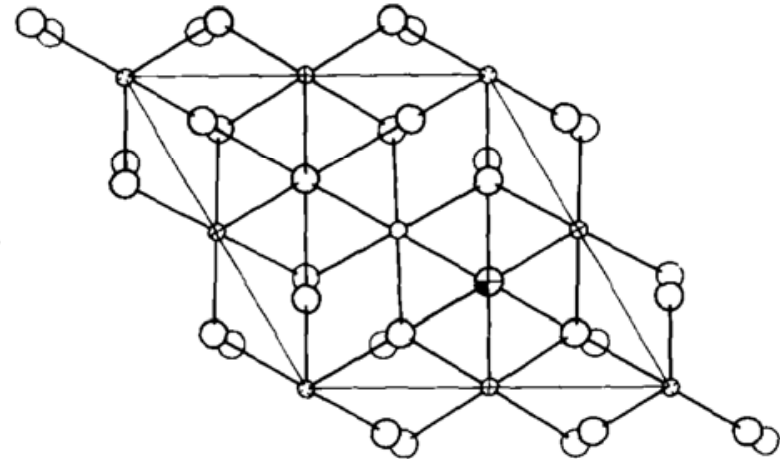

(b)

图 $2 \mathrm{SrNi}_{x} \mathrm{~V}_{6-}{ }_{x} \mathrm{O}_{11}$ 的晶体结构图

(a) 晶胞, 右边显示了原子的层状分布; (b) (001) 方向的投影图, $z$ 值介于 $-0.25 \sim 0.25$, 空心图图为 $\mathrm{O}$ 原子,

带十字圆圈为 $\mathrm{V}(\mathrm{Ni})$ 原子, 部分阴影的圆图为 $\mathrm{Sr}$ 原子

表 $2 \mathrm{SrNi}_{x} \mathrm{~V}_{6-x} \mathrm{O}_{11}(x=0.1)$ 和 $\mathrm{SrV}_{6} \mathrm{O}_{11}$ 的晶胞参数和主要键长数据 ${ }^{\mathrm{a})}$

\begin{tabular}{|c|c|c|c|c|c|}
\hline & $\mathrm{SrNi}_{x} \mathrm{~V}_{6-x} \mathrm{O}_{11}$ & $\mathrm{SrV}_{6} \mathrm{O}_{11}$ & & $\mathrm{Sr} \mathrm{Ni}_{x} \mathrm{~V}_{6-x} \mathrm{O}_{11}$ & $\mathrm{SrV}_{6} \mathrm{O}_{11}$ \\
\hline $\begin{array}{l}\text { 晶胞参数: } a_{0} \\
\text { 键长 } / \mathrm{nm}\end{array}$ & $0.57708(8)$ & $0.57716(1)$ & $\begin{array}{l}\text { 晶胞参数: } c_{0} \\
\text { 键长/nm }\end{array}$ & $1.3084(2)$ & $1.30793(5)$ \\
\hline$M(1)-O(1)$ & $0.1925 \times 4$ & $0.1945 \times 4$ & $\mathrm{M}(3)-\mathrm{O}(2)$ & $0.1913 \times 3$ & $0.1832 \times 3$ \\
\hline $\mathrm{M}(1)-\mathrm{O}(3)$ & $0.2056 \times 6$ & $0.2029 \times 2$ & $\mathrm{M}(3)-\mathrm{O}(3)$ & $0.2066 \times 2$ & $0.2111 \times 2$ \\
\hline $\mathrm{M}(2)-\mathrm{O}(1)$ & $0.1947 \times 3$ & $0.1949 \times 3$ & $\mathrm{Sr}-\mathrm{O}(1)$ & $0.2765 \times 6$ & $0.2697 \times 6$ \\
\hline $\mathrm{M}(2)-\mathrm{O}(2)$ & $0.1964 \times 3$ & $0.2025 \times 3$ & $\mathrm{~S}-\mathrm{O}(2)$ & $0.2895 \times 6$ & $0.2891 \times 6$ \\
\hline
\end{tabular}

a) 键长后的乘数为该键的个数 
离子为五配位, 2 个 $O(3)$ 和 3 个 $O(2)$ 构成两个共底的四面体; 该位置易分裂成两个四面体位 置, $O(3)$ 连线垂直于 $O(2)$ 所构成的平面. $\mathrm{Sr}$ 与 6 个 $\mathrm{O}(1)$ 和 6 个 $\mathrm{O}(2)$ 形成配位多面体.

\section{3 离子价态}

为了确定各晶格位置离子的价态, 采用键价方法 (bond-valence method) 计算成键原子间 的价态 $V_{i j}$, 再据此计算各晶格位的价态 $\sum V_{i j}$. 键价参数依据 Brese 等 ${ }^{[8]}$ 提供的值, 计算了 $\mathrm{SrV}_{6} \mathrm{O}_{11}, \mathrm{NaV}_{6} \mathrm{O}_{11}$ 和 $\mathrm{SrNi}_{x} \mathrm{~V}_{6-x} \mathrm{O}_{11}(x=0.1)$ 各晶格位的价态, 结果列于表 3. $\mathrm{SrV}_{6} \mathrm{O}_{11}$, $\mathrm{NaV}_{6} \mathrm{O}_{11}$ 的晶体结构数据取自文献 [6].

表 3 键价方法计算的各晶格位的价态

\begin{tabular}{|c|c|c|c|c|c|c|}
\hline \multirow{2}{*}{ 原子 } & \multicolumn{2}{|c|}{$\mathrm{SrV}_{6} \mathrm{O}_{11}$} & \multicolumn{2}{|c|}{$\mathrm{NaV}_{6} \mathrm{O}_{11}$} & \multicolumn{2}{|c|}{$\mathrm{SrNi}_{x} \mathrm{~V}_{6-}{ }_{x} \mathrm{O}_{11}$} \\
\hline & $\left.{ }^{3+} \sum V_{i j}{ }^{0}\right)$ & ${ }^{4+} \sum V_{i j}$ & ${ }^{3+} \sum V_{i j}$ & ${ }^{4+} \sum V_{i j}$ & ${ }^{3+} \sum V_{i j}$ & ${ }^{4+} \Sigma V_{i j}$ \\
\hline$M(1)$ & 3.24 & 3.62 & 3.22 & 3.58 & 3.30 & 3.69 \\
\hline $\mathrm{M}(2)$ & 3.11 & 3.48 & 3.37 & 3.76 & 3.38 & 3.78 \\
\hline$M(3)$ & 2.92 & 3.25 & 3.46 & 3.86 & 2.73 & 3.05 \\
\hline$O(1)$ & 1.94 & 2.14 & 1.90 & 2.11 & 1.97 & 2.18 \\
\hline$O(2)$ & 1.96 & 2.17 & 1.95 & 2.16 & 1.85 & 2.18 \\
\hline $\mathrm{O}(3)$ & 1.76 & 1.96 & 1.77 & 1.98 & 1.71 & 1.91 \\
\hline $\mathrm{Sr} / \mathrm{Na}$ & \multicolumn{2}{|c|}{2.00} & \multicolumn{2}{|c|}{0.81} & \multicolumn{2}{|c|}{1.78} \\
\hline
\end{tabular}

a) ${ }^{3+} \sum V_{i j}$ 表示该栏的价态是假定 $\mathrm{M}$ 位完全由 $3+$ 离子占据而计算的结果

由表 3 看出, 任何 $\mathrm{M}$ 位置都不会是由单一价态的离子占有. 对 $\mathrm{SrV}_{6} \mathrm{O}_{11}, \mathrm{SrNi}_{x} \mathrm{~V}_{6-} \mathrm{O}_{11}$, $M(1), M(2)$ 位倾向于由 $3+$ 和 $4+$ 离子共同占有, 而不是由 Madelung 能量法所计算的 $M(1)$ 位由 $3+$ 离子占有, 而 $M(2)$ 位由 $4+$ 离子占有 ${ }^{[6]} ; M(1)$ 位和 $M(2)$ 位较低的温度因子值也反 映这一事实. 对 $\mathrm{SrV}_{6} \mathrm{O}_{11}$, 其 $\mathrm{M}(1)$ 位的 $4+$ 离子占有率较 3+ 高, 而 $\mathrm{SrNi}_{x} \mathrm{~V}_{6-} \mathrm{O}_{11}$ 中, $\mathrm{M}(2)$ 位 则以 $4+$ 离子为主. 因此 $\mathrm{V}$ 离子被 $\mathrm{Ni}$ 离子取代后, 会影响该类化合物中的价态分布. 但两者 的 $\mathrm{M}(3)$ 位均倾向于主要由 $3+$ 离子占有. 在 $\mathrm{SrNi}_{x} \mathrm{~V}_{6-{ }_{x}} \mathrm{O}_{11}, \mathrm{SrFeV}_{5} \mathrm{O}_{11}$ 和 $\mathrm{SrTi}_{x} \mathrm{~V}_{6-{ }_{x}} \mathrm{O}_{11}$ 中, $\mathrm{Ni}^{3+}$ 和 $\mathrm{Fe}^{3+}$ 离子较多地进入 $\mathrm{M}(3)$ 位, $\mathrm{Ti}^{4+}$ 离子较多地进入 $\mathrm{M}(2)$ 位 ${ }^{[5]}$ 也可以说明这一点. 在 $\mathrm{A}$ 位为一价 $\mathrm{Na}$ 离子的 $\mathrm{NaV}_{6} \mathrm{O}_{11}$ 中, $\mathrm{M}(1$ ) 倾向于主要由 $3+$ 离子占据, $\mathrm{M}(2)$ 和 $\mathrm{M}(3)$ 则主要由 $4+$ 离子占据, 这与文献结果是相似的. 当然, 具体离子进入晶格中的位置, 还会受其离子大小 和外围电子构型的影响.

\section{3 结论}

在 $\mathrm{SrV}_{6} \mathrm{O}_{11}$ 中, $\mathrm{M}(3)$ 位主要由 $3+$ 离子占据; $\mathrm{M}(1)$ 和 $\mathrm{M}(2)$ 位由 $3+$ 和 $4+$ 离子混合占据, 其中 $\mathrm{M}(1)$ 位中 $4+$ 离子的含量相对高. 取代 $\mathrm{Ni}$ 离子优先占据 $\mathrm{M}(3)$ 位置; 同时 $\mathrm{M}(1)$ 和 $\mathrm{M}(2)$ 位置的 $3+$ 和 $4+$ 离子的相对含量也发生了变化.

致谢 感谢美国 Georgia 理工学院 R. A. Young 教授提供 DBWS 9411 程序, 克罗地亚Zagreb 大学 Ivan Vickovic 教授赠送 ORTEP 92 软件,杭州大学吕光烈教授为实验数据收集提供了方 便.

\section{参考文献}

1 Kanke Y, Takayama-Muromachi E, Kato K, et al. Phase equilibrium study of the system $\mathrm{Na}_{2} \mathrm{~V}_{2} \mathrm{O}_{5}-\mathrm{V}_{2} \mathrm{O}_{3}-\mathrm{V}_{2} \mathrm{O}_{5}$ at $923 \mathrm{~K}$. J Solid State Chem, 1990, 89: 130 137 
【研究简报】

\title{
激光诱发巴基管向金刚石的相转变
}

\author{
张继红 魏秉庆 梁 吉 刘文今 高志栋 吴德海 \\ （清华大学机械工程系, 北京 100084)
}

\section{关钺词 巴基管 金刚石 激光 扫描电子显微镜 Raman 光谱}

金刚石由于其超高的硬度, 已逐渐成为切削、抗磨等方面的首选材料. $\mathrm{C}_{60}$ 发现后, 已有利 用冲击法将其转变成金刚石的报道 ${ }^{[1]}$. 巴基管与 $\mathrm{C}_{60}$ 结构上类似 ${ }^{[2,3]}$, 但它相对于 $\mathrm{C}_{60}$ 来讲, 成本低、容易大量生产 ${ }^{[4]}$, 因此更有可能进入工程应用. 目前, 利用巴基管作为衬底, 通过化 学气相沉积的方法已生长出良好的金刚石薄膜, 并使金刚石的成核和生长速率大为提高 ${ }^{[5]}$. 同时, 巴基管在高温高压下合成金刚石 ${ }^{[6]}$ 的研究工作也取得了可喜的进展. 本文以球墨铸铁 为基体, 以巴基管为涂层, 通过激光熔凝和后续热处理的方法, 在铁-碳合金体系中获得了微米 尺寸的金刚石晶粒.

\section{1 实验}

\section{1 样品的制备}

纯度为 $50 \%$ 左右的巴基管由石墨-电弧法制备, 经过粉碎、过笁, 粒度在 $80 \mu \mathrm{m}$ 以下．然 后, 将其均匀涂覆在基体表面, 涂层厚度 $0.2 \sim 0.3 \mathrm{~mm}$. 基体选用 $30 \mathrm{~mm} \times 10 \mathrm{~mm} \times 10 \mathrm{~mm}$ 的 珠光体球墨铸铁, 其中, 珠光体含量 $85 \% \sim 90 \%$, 铁素体 $10 \% \sim 15 \%$. 其化学成分 (\%) 为: C: $3.3 \sim 3.6, \mathrm{Si}: 1.9 \sim 2.4, \mathrm{Cu}: 0.62 \sim 0.77, \mathrm{Mn}:<0.3, \mathrm{P}:<0.07, \mathrm{~S}:<0.02$.

$3 \mathrm{~kW} \mathrm{CO} 2$ 激光器作为热源, 激光功率密度 $10^{4} \mathrm{~W} / \mathrm{cm}^{2}$ 左右, 扫描速度 $500 \sim 1500 \mathrm{~mm} /$ $\min$, 搭接率 $30 \%$. 试样经过激光辐照后, 于 $800 \sim 900{ }^{\circ} \mathrm{C}$ 条件下保温 $10 \sim 15 \mathrm{~min}$, 然后进行淬 火处理.

\section{2 检测仪器}

使用 JSM-43CF 型扫描电子显微镜(SEM)观察试样的组织形貌, 波谱仪分析组织成分,

2 Uchida Y, Kanke Y, Takayama-Muromachi E, et al. Magnetic properties of $\mathrm{NaV}_{6} \mathrm{O}_{11}$. J Phys Soc Jpn, 1991, 60:2 530 2533

3 Uchida Y, Kanke Y, Onoda Y. In: Proc 6th Inter Conf on Ferrites, Tokyo, 1992. 722 724

4 De Roy M E, Besse J P. Chevalier R, et al. Synthese et structure cristalline de $\mathrm{NaV}_{6} \mathrm{O}_{11}$. J Solid State Chem, 1987. 67: 185 $\sim 187$

5 Kanke $\mathrm{Y}$, Izumi F, Takayama-Muromachi $\mathrm{E}$, et al., Synthesis and structure refinement of $\mathrm{AT}_{x} \mathrm{~V}_{6-x} \mathrm{O}_{11}(\mathrm{~T}=\mathrm{Ti}, \mathrm{Cr}$ and Fe). J Solid State Chem, 1991, 92: 261 272

6 Kanke Y, Kato K, Takayama-Muromachi E, et al. Structures of $\mathrm{SrV}_{6} \mathrm{O}_{11}$ and $\mathrm{NaV}_{6} \mathrm{O}_{11}$. Acta Cryst, 1992, C48: 1376 1380

7 Kanke Y, Izumi F, Morii Y, et al. Structure phase transitions in the ferromagnetic vanadium oxide $\mathrm{NaV}_{6} \mathrm{O}_{11}$. J Solid State Chem, 1994, 112: 429 437

8 Brese N E, O'Keeffe M. Bond-valence parameters for solids. Acta Cryst, 1991, B47: 192 